\title{
Highly regioselective tandem formal substitution and decarboxylation of 2-acyl-1- chlorocyclopropanecarboxylates with sodium sulfinates
}

\author{
Yuequan Zhu, and Yuefa Gong * \\ School of Chemistry and Chemical Engineering, Huazhong University of Science and Technology, Wuhan 430074, People's Republic of China
}

\section{ARTICLE INFO}

\section{Article history:}

Received

Received in revised form

Accepted

Available online

Keywords:

Tandem reaction

Decarboxylation

Sodium sulfinate

Cyclopropene

\section{ABSTRACT}

This article reported a highly regioselective tandem formal nucleophilic substitution and decarboxylation reaction of alkyl 2-acyl-1-chlorocyclopropanecarboxylates with sodium sulfinates under basic conditions. Actually, alkyl 2-acyl-1-chlorocyclopropanecarboxylates could be easily converted into cyclopropene intermediates by simple 1,2-elimination of hydrogen chloride, and this highly reactive cyclopropene quickly combines with sodium sulfinate via a regioselective 1,4-Michael addition. Subsequent esterolysis and decarboxylation of the 1,4-Michael adducts afforded 1-aroylsulfonyl-2-aroylcyclopropanes in high yields. This observation firstly demonstrates that direct Michael addition of sodium sulfinates with reactive cyclopropene is really workable.

2016 Elsevier Ltd. All rights reserved.

\section{Introduction}

Cyclopropenes, for their unique reactivity that extends far beyond chemical properties typical for common alkenes, has attracted increasing attention of chemists in the past decades. ${ }^{1}$ Recently, a more reactive cyclopropene intermediate I (Scheme $1, \mathrm{~A})$ bearing two electron-withdrawing groups on its $\mathrm{C}=\mathrm{C}$ bond was observed during our investigation in the formal fluorination reaction of ethyl 2-aroyl-1-chlorocyclopropanecarboxylates with $\mathrm{HF}_{2}^{-}$or $\mathrm{H}_{2} \mathrm{~F}_{3}{ }^{-2}$. To our knowledge, this is one successful example for unique nucleophilic addition reaction of hydrogen fluoride with electro-deficient alkenes under weakly basic conditions.

Inspired by this observation, we want to evaluate nucleophilic addition reactions of other typical weak nucleophiles with the electron-deficient cyclopropenes generated in situ. Among them, sodium sulfinates as a kind of weaker nucleophile, to date, are rarely reported in direct Michael addition reaction with electrondeficient alkenes except with some special olefines such as alkenyliodonium salt (Scheme 1, B) ${ }^{3}$ For the wide applications of cyclopropyl sulfones in synthesis ${ }^{4-8}$ and potential antimicrobial and pesticidal activitives, ${ }^{9}$ our attention was focused on the prossibility to prepare cyclopropyl sulfones through direct ThiaMichael addition of sodium sulfinates with the reactive electrondeficient cyclopropenes (Scheme 1, C).

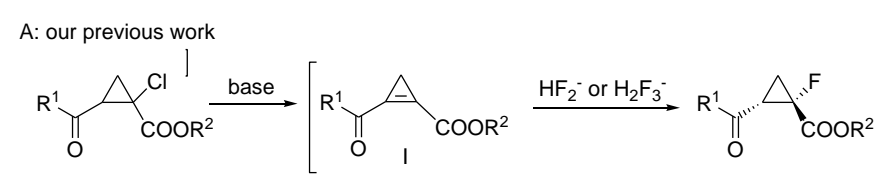

B: previous work

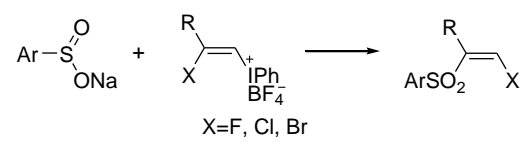

C: proposed reaction in this work

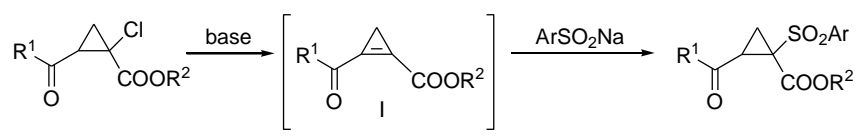

Scheme 1. The additive reactions of weak nucleophiles with alkenes.

In general, cyclopropyl sulfones could be prepared from the cycloaddition of vinylic sulfones with active methylene compounds, ${ }^{10}$ cyclization of sulfone-stabilized carbanions and electron-deficient alkenes, ${ }^{11}$ or cycloisomerization of terminal epoxides with sodium salts of diethyl (phenylsulfonyl)methylphosphonate. ${ }^{12}$ Moreover, they could also be formed from further oxidation of cyclopropyl sulfides. ${ }^{13}$ There is still a great demand for a general and convenient procedure from readily available starting materials.

Based on the above idea and current research situation, we have tried the possible reaction between 2-aroyl-1-

\footnotetext{
* Corresponding author. Tel.: +86 027 87543232; fax: +86 027 87543632; e-mail: gongyf@ mail.hust.edu.cn.
} 
chlorocyclopropanecarboxylates and sodium sulfinates. As a consequence, a highly regioselective tandem reaction involving 1,2-elimination of $\mathrm{HCl}, 1,4-$ Michael addition of arylsulfinate, esterolysis and decarboxylation occurred smoothly, affording a variety of cyclopropyl sulfones in high yields with $d r>20: 1$. In fact, it was the first instance reported hitherto about direct Michael addition of sodium arylsulfinate with cyclopropene intermediate I. Moreover, the introduction of sulfonyl group makes the subsequent esterolysis and decarboxylation proceed more easily in a tandem process.

\section{Results and Discussion}

Initially, we began our study choosing ethyl 2-benzoyl-1chlorocyclopropanecarboxylate 1a and sodium benzenesulfinate 2a as benchmark substrates. Fortunately, the product 4aa in $74 \%$ yield with $d r>20: 1$ was observed in the presence of 1 equiv. $\mathrm{Cs}_{2} \mathrm{CO}_{3}$ in DMSO at $80^{\circ} \mathrm{C}$ (Scheme 2). The structure of product Table 1

Optimization of reaction conditions ${ }^{\mathrm{a}}$.

\begin{tabular}{|c|c|c|c|c|c|c|}
\hline \multirow{2}{*}{ entry } & \multirow{2}{*}{ base } & \multirow{2}{*}{ solvent } & \multirow{2}{*}{ time $(h)^{b}$} & \multirow{2}{*}{ conversion $(\%)$} & \multicolumn{2}{|c|}{$\operatorname{yield}(\%)^{\mathrm{c}}$} \\
\hline & & & & & 3aa & 4aa \\
\hline 1 & $\mathrm{~K}_{2} \mathrm{CO}_{3}$ & DMSO & 24 & 62 & $62(38)$ & $16(10)$ \\
\hline 2 & $\mathrm{Na}_{2} \mathrm{CO}_{3}$ & DMSO & 24 & 40 & $64(26)$ & $12(5)$ \\
\hline 3 & $\mathrm{~K}_{3} \mathrm{PO}_{4}$ & DMSO & 10 & 100 & 19 & 70 \\
\hline 4 & $\mathrm{Cs}_{2} \mathrm{CO}_{3}$ & DMSO & 2 & 100 & - & 89 \\
\hline 5 & $\mathrm{NaOH}$ & DMSO & 5 & 100 & 10 & 48 \\
\hline 6 & $\mathrm{KOH}$ & DMSO & 4 & 100 & 4 & 48 \\
\hline 7 & $\mathrm{CH}_{3} \mathrm{ONa}$ & DMSO & 3 & 100 & 16 & 33 \\
\hline 8 & t-BuOK & DMSO & 0.5 & 100 & - & 26 \\
\hline 9 & DIPEA & DMSO & 24 & 26 & $27(7)$ & - \\
\hline 10 & TEA & DMSO & 24 & 44 & $51(22)$ & - \\
\hline 11 & $\mathrm{DBU}$ & DMSO & 2 & 100 & 16 & 43 \\
\hline 12 & $\mathrm{Cs}_{2} \mathrm{CO}_{3}$ & DMF & 4 & 100 & 11 & 74 \\
\hline 13 & $\mathrm{Cs}_{2} \mathrm{CO}_{3}$ & $\mathrm{CH}_{3} \mathrm{CN}$ & 4 & 100 & 4 & 37 \\
\hline 14 & $\mathrm{Cs}_{2} \mathrm{CO}_{3}$ & 1,4-Dioxane & 24 & 100 & 17 & 15 \\
\hline 15 & $\mathrm{Cs}_{2} \mathrm{CO}_{3}$ & 1,2-DCE & 24 & 46 & - & $43(20)$ \\
\hline 16 & $\mathrm{Cs}_{2} \mathrm{CO}_{3}$ & toluene & 24 & 30 & - & $38(11)$ \\
\hline $17^{\mathrm{d}}$ & $\mathrm{Cs}_{2} \mathrm{CO}_{3}$ & DMSO & 10 & 100 & 9 & 81 \\
\hline $18^{\mathrm{e}}$ & $\mathrm{Cs}_{2} \mathrm{CO}_{3}$ & DMSO & 24 & 100 & 34 & 5 \\
\hline
\end{tabular}

${ }^{\mathrm{a}}$ Unless otherwise noted, the reaction was performed with $\mathbf{1 a}(0.2 \mathrm{mmol}), \mathbf{2 a}(0.4 \mathrm{mmol})$, and the indicated base in solvent at $80^{\circ} \mathrm{c}$.

${ }^{\mathrm{b}}$ Determined by TLC.

${ }^{c}$ Yields based on converted 1a, and isolated yields in parentheses.

${ }^{\mathrm{d}} \mathrm{Cs}_{2} \mathrm{CO}_{3}(0.3 \mathrm{mmol})$.

${ }^{\mathrm{e}}$ Reaction was performed at room temperature.

In order to get the optimal reaction conditions, several parameters including base, solvent and reaction temperature were screened using the above reaction of $\mathbf{1 a}$ with $\mathbf{2 a}$ as the model. The observed results are summarized in Table 1. Firstly, a variety of inorganic and organic bases were assessed. From the results listed in Table 1 (entries 2-11), we can conclude all of the inorganic bases can promote this reaction, but their property has a great influence on the reaction. Common bases like $\mathrm{K}_{2} \mathrm{CO}_{3}$ or $\mathrm{Na}_{2} \mathrm{CO}_{3}$ showed inferior catalytic activity, and gave only low conversions after $24 \mathrm{~h}$ (Table 1, entries 1-2). Strong base was conducive to the further decarboxylation of this reaction process. Among all the inorganic bases such as $\mathrm{K}_{3} \mathrm{PO}_{4}, \mathrm{NaOH}, \mathrm{KOH}$, and $\mathrm{CH}_{3} \mathrm{ONa}, \mathrm{Cs}_{2} \mathrm{CO}_{3}$ was the most suitable one, and the product 4aa was produced in $89 \%$ yield with $d r>20: 1$ possibly owing to its for this process was carefully characterized by ${ }^{1} \mathrm{H}$ and ${ }^{13} \mathrm{C} \mathrm{NMR}$ spectroscopy and HRMS, and the major isomer was assigned to be trans-4aa. This assignment was further supported by single crystal X-ray analysis of product 4 bd (see supporting information). It should be mentioned that product 3aa (in $18 \%$ yield) yielded through simple sulfonate addition, which was hardly separated from 4aa by silica gel column chromatography.

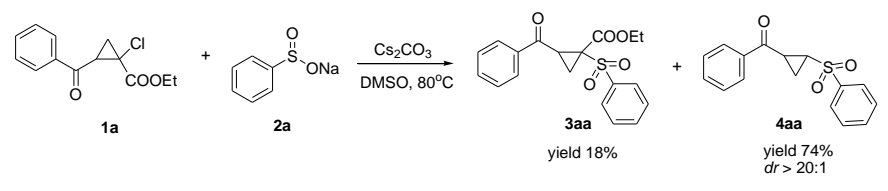

Scheme 2. Formal substitution reaction of 1a with sodium sulfinate 2a. 
Decreasing the loading of base to 1.5 equiv. or lowering reaction temperature resulted in the formation of $4 \mathbf{a a}$ in relatively low yields (Table 1, entries 17-18). Based on the above observations, the optimized reaction conditions chosen for further investigation were as follows: substrate (1 equiv.), sodium sulfinate (2 equiv.), $\mathrm{Cs}_{2} \mathrm{CO}_{3}$ (2 equiv.) in DMSO at $80{ }^{\circ} \mathrm{C}$ (Table 1, entry 4).

Under the optimized conditions, the reactions of sodium benzenesulfinate 2a with a variety of alkyl 2-acyl-1chlorocyclopropanecarboxylates 1a-1o were studied. All the observed results were summarized in Table 2 . In all the cases, the corresponding products $\mathbf{4 b a}-\mathbf{4 k a}$ were respectively produced with excellent diastereoselectivityies ( $d r$ up to 20:1). In contrast, the product yields obviously varied with the change of $\mathrm{R}^{1}$ groups for $\mathbf{1}$, though the effect of their electronic property seems to be irregular (entries 1-10). For example, both 1b with electrondonating 4-Me group and 1d with electron-withdrawing 4-Cl group gave the corresponding products $4 \mathbf{b a}$ and $4 \mathbf{d a}$ in excellent yields, whereas 1c with 4-MeO group and $\mathbf{1 e}$ with $4-\mathrm{Br}$ group provided moderate yields of $\mathbf{4} \mathbf{c a}$ and 4 ea. Among them, the lowest yield was observed in the case of $\mathbf{1 g}$ with a 4-Ph group (entry 7). In addition, substrates $\mathbf{1 h}-\mathbf{1 k}$ with 2-furyl, 2-thienyl, 1naphthyl or 1-pyrenyl groups were also tolerated in this process, affording almost the corresponding products 4ga-4ka in moderate yields with high $d r$ values (up to 20:1), respectively (Table 2, entries 8-11). Moreover, it should be noted that the electronic property of $\mathrm{R}^{1}$ groups of $\mathbf{1}$ has an obvious influence on the reaction rates, and the electron-donating group decelerated the reaction. By the way, the steric effect of $R^{2}$ group on this reaction was also examined under the same conditions. According to the results listed in Table 2 (entries 12-15), we realized that the steric hindrance had a little influence on this cascade process. In fact, both small methyl ester $\mathbf{1 l}$ and the bulky t-butyl ester 1m gave the product 4aa in highest yields in $89 \%$, $87 \%$ with excellent $d r$ value (entries 13-14). When $\mathrm{R}^{2}$ is a functional group like $\left(\mathrm{CH}_{2}\right)_{2} \mathrm{Cl}$ or benzyl, substrates $\mathbf{1 n}$ or $\mathbf{1 0}$ were also adapted to the reaction, furnishing the expected product 4aa in high yields (Table 2, entries 15-16).

Table 2

Substrate scope for the reaction of $\mathbf{1}$ with $\mathbf{2} \mathbf{a a}^{\mathrm{a}}$

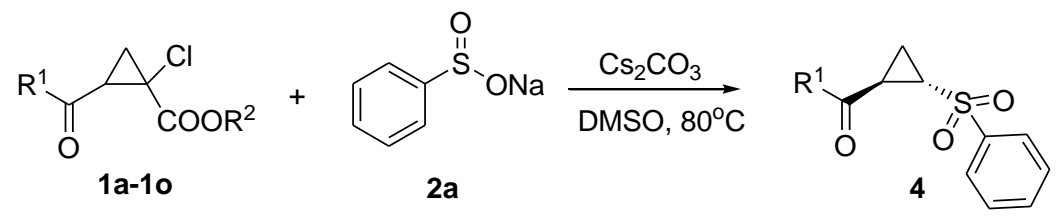

\begin{tabular}{llllllll}
\hline entry & $\mathbf{1}$ & $\mathrm{R}^{1}$ & $\mathrm{R}^{2}$ & time $(\mathrm{h})^{\mathrm{b}}$ & product & yield $(\%)^{\mathrm{c}}$ & $d r^{\mathrm{d}}$ \\
\hline 1 & $\mathbf{1 a}$ & $\mathrm{C}_{6} \mathrm{H}_{5}$ & $\mathrm{Et}$ & 2 & $\mathbf{4 a a}$ & 89 & $>20: 1$ \\
2 & $\mathbf{1 b}$ & $4-\mathrm{MeC}_{6} \mathrm{H}_{4}$ & $\mathrm{Et}$ & 6 & $\mathbf{4 b a}$ & 80 & $>20: 1$ \\
3 & $\mathbf{1 c}$ & $4-\mathrm{MeOC}_{6} \mathrm{H}_{4}$ & $\mathrm{Et}$ & 8 & $\mathbf{4 c a}$ & 68 & $>20: 1$ \\
4 & $\mathbf{1 d}$ & $4-\mathrm{ClC}_{6} \mathrm{H}_{4}$ & $\mathrm{Et}$ & 2 & $\mathbf{4 d a}$ & 88 & $>20: 1$ \\
5 & $\mathbf{1 e}$ & $4-\mathrm{BrC}_{6} \mathrm{H}_{4}$ & $\mathrm{Et}$ & 2 & $\mathbf{4 e a}$ & 60 & $>20: 1$ \\
6 & $\mathbf{1 f}$ & $2-\mathrm{BrC}_{6} \mathrm{H}_{4}$ & $\mathrm{Et}$ & 2 & $\mathbf{4 f a}$ & 48 & $>20: 1$ \\
7 & $\mathbf{1 g}$ & $4-\mathrm{PhC}_{6} \mathrm{H}_{4}$ & $\mathrm{Et}$ & 3 & $\mathbf{4 g a}$ & 33 & $>20: 1$ \\
8 & $\mathbf{1 h}$ & $2-\mathrm{furyl}$ & $\mathrm{Et}$ & 2 & $\mathbf{4 h a}$ & 78 & $>20: 1$ \\
9 & $\mathbf{1 i}$ & $2-$ thienyl & $\mathrm{Et}$ & 5 & $\mathbf{4 i a}$ & 70 & $>20: 1$ \\
10 & $\mathbf{1 j}$ & $1-\mathrm{naphthyl}_{1}$ & $\mathrm{Et}$ & 6 & $\mathbf{4 j a}$ & 55 & $>20: 1$ \\
11 & $\mathbf{1 k}$ & $1-\mathrm{pyrenyl}_{1}$ & $\mathrm{Me}$ & 4 & $\mathbf{4 k a}$ & 72 & $>20: 1$ \\
12 & $\mathbf{1 l}$ & $\mathrm{C}_{6} \mathrm{H}_{5}$ & $\mathrm{Me}$ & 2 & $\mathbf{4 a a}$ & 89 & $>20: 1$ \\
13 & $\mathbf{1 m}$ & $\mathrm{C}_{6} \mathrm{H}_{5}$ & $\mathrm{t}-\mathrm{Bu}$ & 8 & $\mathbf{4 a a}$ & 87 & $>20: 1$ \\
14 & $\mathbf{1 n}$ & $\mathrm{C}_{6} \mathrm{H}_{5}$ & $(\mathrm{CH})_{2} \mathrm{Cl}$ & 4 & $\mathbf{4 a a}$ & 79 & $>20: 1$ \\
15 & $\mathbf{1 0}$ & $\mathrm{C}_{6} \mathrm{H}_{5}$ & $\mathrm{Benzyl}$ & 2 & $\mathbf{4 a a}$ & 73 & $>20: 1$
\end{tabular}

${ }^{\mathrm{a}}$ Reaction conditions: $\mathbf{1}(0.2 \mathrm{mmol}), \mathbf{2 a}(0.4 \mathrm{mmol})$ and $\mathrm{Cs}_{2} \mathrm{CO}_{3}(0.4 \mathrm{mmol})$ in DMSO $(2 \mathrm{~mL})$ was stirred at $80^{\circ} \mathrm{C}$.

${ }^{b}$ Determined by TLC.

${ }^{\mathrm{c}}$ Isolated by column chromatography.

${ }^{\mathrm{d}}$ Diastereomeric ratios (trans:cis) determined by ${ }^{1} \mathrm{H}$ NMR of the crude product.

Next, we examined the reaction of 2-benzoyl-1chlorocyclopropaneformate $\mathbf{1 a}$ with a group of sodium sulfinates. And the results were summarized in Table 3 . The introduction of electronically different substituents such as $\mathrm{Me}, \mathrm{OMe}$ and $\mathrm{Cl}$ groups on the para position of benzene ring of sodium benzenesulfinate caused a marked decrease in the yields of the expected products $\mathbf{4 a b}$, 4ac and 4ad, despite of the high $d r$ values (Table 3, entries 2-4). Sodium thiophene-2-sulfinate $\mathbf{2 e}$ can also take reaction with 1a smoothly to give the expected product 4ae in 68\% yield with $d r>20: 1$ (Table 3, entry 5). In the case of sodium methanesulfinate $\mathbf{2 f}$, the reaction also underwent smoothly and afforded the formal substitution product 3af with high $d r$ value (Table 3, entry 6) rather than the desired decarboxylation product, indicative that the property of sulfonyl group has a big influence on the subsequent esterolysis. Sodium trifluoromethanesulfinate $\mathbf{2 g}$, a much weaker nucleophile, did not 
react with 1a under the same conditions (Table 3, entry 7). Similar substituent effect of $\mathrm{R}^{1}$ group of $\mathbf{1}$ on the reaction rate and the product yields was also observed in these cases (Table 3, entries 8-13).

Table 3

Substrate scope for the reaction of $\mathbf{1}$ with $\mathbf{2}^{\mathrm{a}}$

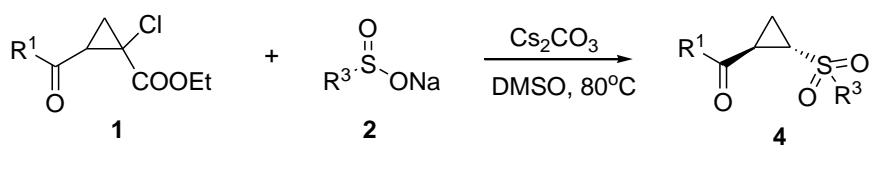

\begin{tabular}{lllllll}
\hline entry & $\mathbf{1}$ & $\mathbf{2}$ & $\begin{array}{l}\text { time } \\
(\mathrm{h})^{\mathrm{b}}\end{array}$ & product & $\begin{array}{l}\text { yield } \\
(\%)^{\mathrm{c}}\end{array}$ & $d r^{\mathrm{d}}$ \\
\hline 1 & $\mathbf{1 a}$ & $\mathbf{2 a}\left(\mathrm{R}^{3}=\mathrm{C}_{6} \mathrm{H}_{5}\right)$ & 2 & $\mathbf{4 a a}$ & 89 & $>20: 1$ \\
2 & $\mathbf{1 a}$ & $\mathbf{2 b}\left(\mathrm{R}^{3}=4-\mathrm{MeC}_{6} \mathrm{H}_{4}\right)$ & 9 & $\mathbf{4 a b}$ & 50 & $>20: 1$ \\
$3^{[\mathrm{e}]}$ & $\mathbf{1 a}$ & $\mathbf{2 c}\left(\mathrm{R}^{3}=4-\mathrm{OMeC}_{6} \mathrm{H}_{4}\right)$ & 7 & $\mathbf{4 a c}$ & 66 & $>20: 1$ \\
4 & $\mathbf{1 a}$ & $\mathbf{2 d}\left(\mathrm{R}^{3}=4-\mathrm{ClC}_{6} \mathrm{H}_{4}\right)$ & 9 & $\mathbf{4 a d}$ & 51 & $>20: 1$ \\
$5^{[\mathrm{e}]}$ & $\mathbf{1 a}$ & $\mathbf{2 e}\left(\mathrm{R}^{3}=2-\right.$-thienyl $)$ & 6 & $\mathbf{4 a e}$ & 68 & $>20: 1$ \\
6 & $\mathbf{1 a}$ & $\mathbf{2 f}\left(\mathrm{R}^{3}=\mathrm{Me}\right)$ & 24 & $\mathbf{3 a f}$ & 53 & $>20: 1$ \\
7 & $\mathbf{1 a}$ & $\mathbf{2 g}\left(\mathrm{R}^{3}=\mathrm{CF}_{3}\right)$ & 24 & $\mathrm{NR}$ & -- & -- \\
8 & $\mathbf{1 b}$ & $\mathbf{2 b}$ & 12 & $\mathbf{4 b b}$ & 51 & $>20: 1$ \\
9 & $\mathbf{1 b}$ & $\mathbf{2 d}$ & 12 & $\mathbf{4 b d}$ & 33 & $>20: 1$ \\
10 & $\mathbf{1 d}$ & $\mathbf{2 b}$ & 5.5 & $\mathbf{4 d b}$ & 63 & $>20: 1$ \\
11 & $\mathbf{1 d}$ & $\mathbf{2 d}$ & 6 & $\mathbf{4 d d}$ & 43 & $>20: 1$ \\
$12^{[\mathrm{e}]}$ & $\mathbf{1 i}$ & $\mathbf{2 b}$ & 12 & $\mathbf{4 i b}$ & 51 & $>20: 1$ \\
$13^{[\mathrm{e}]}$ & $\mathbf{1 i}$ & $\mathbf{2 d}$ & 12 & $\mathbf{4 i d}$ & 19 & $>20: 1$ \\
\hline
\end{tabular}

${ }^{\mathrm{a}}$ Reaction conditions: $\mathbf{1}(0.2 \mathrm{mmol}), \mathbf{2 a}(0.4 \mathrm{mmol})$ and $\mathrm{Cs}_{2} \mathrm{CO}_{3}(0.4 \mathrm{mmol})$ in DMSO $(2 \mathrm{~mL})$ was stirred at $80{ }^{\circ} \mathrm{C}$.

${ }^{\mathrm{b}}$ Determined by TLC.

'Isolated by column chromatography.

${ }^{\mathrm{d}}$ Diastereomeric ratios (trans:cis) determined by ${ }^{1} \mathrm{H}$ NMR of the crude product.

${ }^{\mathrm{e}} \mathrm{Cs}_{2} \mathrm{CO}_{3}(0.5 \mathrm{mmol})$.

In order to further investigate the tandem process, we conducted some control experiments shown in Scheme 3. First, we found that only the formal substitution product 3aa was generated and isolated in $83 \%$ yield when the reaction of $1 \mathbf{a}$ and $2 \mathrm{a}$ was performed in the presence of 1.5 equiv. $\mathrm{Cs}_{2} \mathrm{CO}_{3}$ at room temperature (Scheme 3, a). When the isolated product 3aa was treated with 1 equiv. $\mathrm{Cs}_{2} \mathrm{CO}_{3}$ in DMSO at $80{ }^{\circ} \mathrm{C}$, it was readily converted into $4 \mathbf{a a}$ in 88\% yield with $d r>20: 1$ (Scheme 3, b). In this case, the total yield for $\mathbf{4 a a}$ is $70 \%$. When the crude product 3aa was further treated with additional 1 equiv. of $\mathrm{Cs}_{2} \mathrm{CO}_{3}$ in DMSO at $80{ }^{\circ} \mathrm{C}$ without isolation, the desired decarboxylation product 4aa was obtained in total $66 \%$ yield (Scheme 3, c). Compared with the $89 \%$ yield (Table 2, entry 1 ), the relatively lower yields imply that the simultaneous esterolysis and decarboxylation would benefit the formal substitution reaction to some extent. In addition, the existence of arylsulfonyl group made the esterolysis and decarboxylation of 3aa proceed more easily in basic media.

According to the above investigation, a possible reaction mechanism was proposed and depicted in Scheme 4. The reaction was initiated by a simple 1,2-elimination of $\mathrm{HCl}$, giving a highly reactive cyclopropene intermediate I. Next, sodium arylsulfinate attacked the intermediate I via Michael addition to furnish the formal substitution compound 3aa under basic conditions. Subsequent esterolysis and decarboxylation of 3aa provided the cyclopropyl aryl sulfone 4aa. Though the activation role of arylsulfonyl group is still kept unclear, we presumed that its orientation and coordination ability with cesium ion plays a crucial role in the esterolysis process depicted in Scheme 4, since the analogous methylsulfonyl group did not have the role. In addition, we speculated that stereoelectronic and steric effects between aroyl and sulfonyl groups played the key role for the excellently diastereoselective formation of trans-4aa during the final protonation step as shown in Scheme 4.

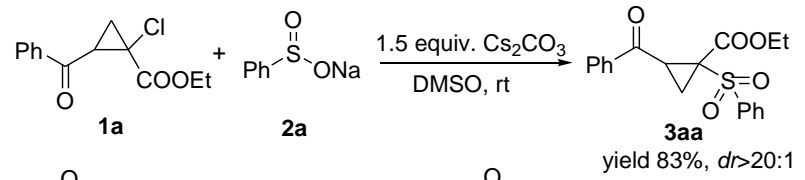

(b)

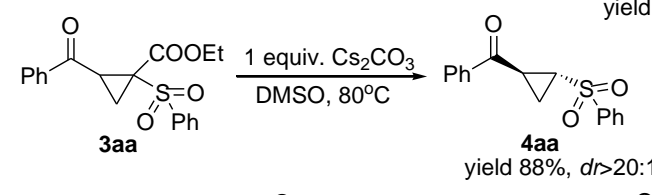

(c)

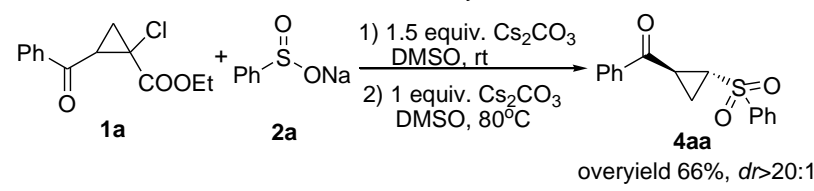

Scheme 3. Some control experiments<smiles>CCOC(=O)C1(Cl)C[C@H]1C(=O)c1ccccc1</smiles>

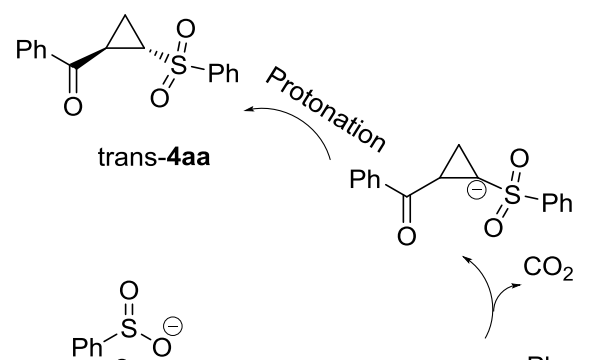<smiles>CCOC(=O)C1CC1C(=O)c1ccccc1</smiles><smiles>O=C([O-])C1OC1(C(=O)c1ccccc1)S(=O)(=O)c1ccccc1</smiles>

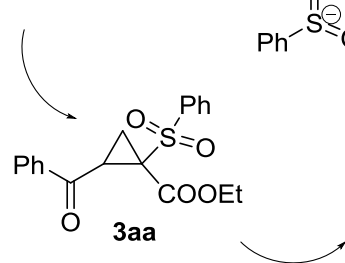<smiles>CCO[C@H](C)OC(=O)C1(S(=O)(=O)c2ccccc2)CC1C(=O)c1ccccc1</smiles>

Scheme 4. Proposed mechanism for the tandem reaction.

\section{Conclusions}

In summary, a novel and metal-free method for the high regioselective addition of the electron-deficient reactive cyclopropene intermediate generated in situ from 2-acyl-1chlorocyclopropanecarboxylate with sodium sulfinate has been developed under mild conditions. A broad range of 1aroylsulfonyl-2-aroylcyclopropane derivatives, which involves the 1,2-elimination/addition/esterolysis/decarboxylation pathway, was obtained in up to $89 \%$ yield with excellent $d r$ value. In addition, the synthetically useful research of this special electrondeficient cyclopropene intermediate generated in situ is currently underway in our laboratory.

\section{Experimental section}

\subsection{General information}

All isolated compounds were characterized on the basis of ${ }^{1} \mathrm{H}$ NMR and ${ }^{13} \mathrm{C}$ NMR spectroscopic data, IR spectra, and HRMS data. All ${ }^{1} \mathrm{H}$ NMR and ${ }^{13} \mathrm{C}$ NMR spectra were recorded on a 400 
$\mathrm{MHz}$ spectrometer with solvent resonances as the internal standard $\left({ }^{1} \mathrm{H}\right.$ NMR: $\mathrm{CDCl}_{3}$ at $7.26 \mathrm{ppm} ;{ }^{13} \mathrm{C} \mathrm{NMR:} \mathrm{CDCl}_{3}$ at 77.0 $\mathrm{ppm})$. Infrared spectra were recorded with a FT-IR spectrometer. High-resolution mass spectra (HRMS) were recorded with a Bruker TOF-Q spectrometer in the EI mode. Melting points were recorded with a melting point detector. All reactions were monitored by TLC analysis using silica gel $60 \AA$ A F-254 thin layer plates and visualized by UV. Flash column chromatography was performed on silica gel $60 \AA, 10-40 \mu \mathrm{m}$. All reagents and solvents were of commercial grade and purified prior to use when necessary. Unless otherwise noted, 2-acyl-1chlorocyclopropanecarboxylate were synthesized according to literature. $^{2}$

\subsection{Typical Procedure for the Synthesis of cyclopropyl aryl sulfones 4:}

Compounds 2-benzoyl-1-chlorocyclopropanecarboxylate 1a (50 mg, $0.2 \mathrm{mmol})$, sodium benzenesulfinate $2 \mathbf{a}(66 \mathrm{mg}, 0.4$ mmol) and $\mathrm{Cs}_{2} \mathrm{CO}_{3}(131 \mathrm{mg}, 0.4 \mathrm{mmol}$ ) were putted into $2 \mathrm{~mL}$ of DMSO at room temperature, and then the reaction mixture was heated and stirred at $80{ }^{\circ} \mathrm{C}$. The reaction was followed by TLC until all the substrate 1a disappeared. The reaction was cooled to room temperature and was diluted with water $(10 \mathrm{~mL})$. Further treatment was followed by extraction with $\mathrm{CH}_{2} \mathrm{Cl}_{2}(2 \times$ $20 \mathrm{~mL}$ ). The combined organic extracts were washed with $\mathrm{H}_{2} \mathrm{O}$ $(20 \mathrm{~mL})$ and brine $(3 \times 20 \mathrm{~mL})$, dried with anhydrous $\mathrm{Na}_{2} \mathrm{SO}_{4}$, filtered, and concentrated under reduced pressure. The residue was purified by silica gel column chromatography using petroleum ether-EtOAc as eluent to afford the corresponding product 4aa. Unless otherwise specified, all other products $\mathbf{3}$ and 4 were obtained according to this typical procedure. All the products 4 with trans configuration prepared in this work are racemic.

\subsection{Phenyl((1S,2R)-2-(phenylsulfonyl)cyclopropyl)methanone (4aa)}

White solid (51 mg, $89 \%$ yield). mp. $120.7-122.3{ }^{\circ} \mathrm{C} .{ }^{1} \mathrm{H}$ NMR (400 MHz, $\mathrm{CDCl}_{3}, 25{ }^{\circ} \mathrm{C}$, TMS): $\delta=7.99$ (d, $J=7.7 \mathrm{~Hz}, 2 \mathrm{H}$, $\operatorname{ArH}), 7.94(\mathrm{~d}, J=7.7 \mathrm{~Hz}, 2 \mathrm{H}, \operatorname{ArH}), 7.66(\mathrm{dd}, J=13.8,6.2 \mathrm{~Hz}$, $1 \mathrm{H}, \mathrm{ArH}), 7.59(\mathrm{dd}, J=15.5,7.5 \mathrm{~Hz}, 3 \mathrm{H}, \mathrm{ArH}), 7.49(\mathrm{t}, J=7.7$ $\mathrm{Hz}, 2 \mathrm{H}, \mathrm{ArH}$ ), 3.52 (ddd, $J=9.7,5.7,4.2 \mathrm{~Hz}, 1 \mathrm{H}, \mathrm{CH}-\mathrm{cyclo}$ ), 3.18 (ddd, $J=9.8,5.8,4.2 \mathrm{~Hz}, 1 \mathrm{H}, \mathrm{CH}$-cyclo), $1.90-1.82(\mathrm{~m}$, $1 \mathrm{H}, \mathrm{CH}_{2}$-cyclo), 1.74 (dt, $J=8.5,5.3 \mathrm{~Hz}, 1 \mathrm{H}, \mathrm{CH}_{2}$-cyclo). ${ }^{13} \mathrm{C}$ NMR (101 MHz, $\left.\mathrm{CDCl}_{3}, 25{ }^{\circ} \mathrm{C}, \mathrm{TMS}\right): \delta=195.2,139.9,136.49$, $133.9,129.5,129.4,128.8,128.4,127.7,42.2,23.0,15.3$. IR (neat): $v$ 3025, 2923, 1731, 1664, 1592, 1447, 1385, 1306, 1266, $1225,1149,1084,1063,1023,981,916,861,794 \mathrm{~cm}^{-1}$. HRMS (EI): $\mathrm{m} / \mathrm{z}$ calcd for $\mathrm{C}_{16} \mathrm{H}_{15} \mathrm{O}_{3} \mathrm{~S} \quad[\mathrm{M}+\mathrm{H}]^{+}$: 287.0742, found: 287.0745 .

\section{4. ((1S,2R)-2-(Phenylsulfonyl)cyclopropyl)(p-tolyl)methanone (4ba)}

Light yellow solid (48 mg, 80\% yield). mp. 129.3-131.1 ${ }^{\circ} \mathrm{C}$. ${ }^{1} \mathrm{H}$ NMR $\left(400 \mathrm{MHz}, \mathrm{CDCl}_{3}, 25{ }^{\circ} \mathrm{C}\right.$, TMS): $\delta=7.97-7.86(\mathrm{~m}$, $4 \mathrm{H}, \mathrm{ArH}), 7.68-7.62(\mathrm{~m}, 1 \mathrm{H}, \mathrm{ArH}), 7.56(\mathrm{dd}, J=10.5,4.7 \mathrm{~Hz}$, $2 \mathrm{H}, \mathrm{ArH}), 7.30-7.26(\mathrm{~m}, 2 \mathrm{H}, \mathrm{ArH}), 3.55-3.44(\mathrm{~m}, 1 \mathrm{H}, \mathrm{CH}-$ cyclo), 3.17 (ddd, $J=8.5,5.8,4.1 \mathrm{~Hz}, 1 \mathrm{H}, \mathrm{CH}$-cyclo), 2.42 (s, $3 \mathrm{H}, \mathrm{CH}_{3}$ ), 1.83 (tt, $J=13.8,6.4 \mathrm{~Hz}, 1 \mathrm{H}, \mathrm{CH}_{2}$-cyclo), 1.72 (ddd, $J$ $=8.5,5.8,4.7 \mathrm{~Hz}, 1 \mathrm{H}, \mathrm{CH}_{2}$-cyclo). ${ }^{13} \mathrm{C} \mathrm{NMR}\left(101 \mathrm{MHz} \mathrm{CDCl}_{3}\right.$, $\left.25{ }^{\circ} \mathrm{C}, \mathrm{TMS}\right): \delta=194.6,144.9,139.89,133.9,133.9,129.5$, $129.5,128.6,127.7,42.1,22.9,21.7,15.2$. IR (neat): $v$ 3027, 2922, 1734, 1662, 1603, 1447, 1418, 1383, 1307, 1233, 1209, 1178, 1148, 1086, 1061, 1033, 985, 920, 859, 817, $748 \mathrm{~cm}^{-1}$. HRMS (EI): $\mathrm{m} / \mathrm{z}$ calcd for $\mathrm{C}_{17} \mathrm{H}_{17} \mathrm{O}_{3} \mathrm{~S}[\mathrm{M}+\mathrm{H}]^{+}: 301.0898$, found: 301.0888 .
4.5. (4-Methoxyphenyl)((1S,2R)-2-(phenylsulfonyl)cyclopropyl)methanone (4ca)

Light yellow solid (43 mg, 68\% yield). mp. 85.6-87.3 ${ }^{\circ} \mathrm{C} .{ }^{1} \mathrm{H}$ NMR (400 MHz, $\left.\mathrm{CDCl}_{3}, 25^{\circ} \mathrm{C}, \mathrm{TMS}\right): \delta=7.97(\mathrm{t}, J=7.5 \mathrm{~Hz}$, $2 \mathrm{H}, \mathrm{ArH}), 7.93(\mathrm{~d}, J=7.2 \mathrm{~Hz}, 2 \mathrm{H}, \mathrm{ArH}), 7.65(\mathrm{t}, J=7.4 \mathrm{~Hz}, 1 \mathrm{H}$, $\operatorname{ArH}), 7.57(\mathrm{t}, J=7.6 \mathrm{~Hz}, 2 \mathrm{H}, \operatorname{ArH}), 6.95(\mathrm{~d}, J=8.9 \mathrm{~Hz}, 2 \mathrm{H}$, $\mathrm{ArH}), 3.88$ (s, 3H, $\left.\mathrm{OCH}_{3}\right), 3.51-3.43$ (m, 1H, CH-cyclo), 3.15 (ddd, $J=8.6,5.8,4.2 \mathrm{~Hz}, 1 \mathrm{H}, \mathrm{CH}$-cyclo), $1.86-1.77(\mathrm{~m}, 1 \mathrm{H}$, $\mathrm{CH}_{2}$-cyclo), 1.71 (dt, $J=8.5,5.2 \mathrm{~Hz}, 1 \mathrm{H}, \mathrm{CH}_{2}$-cyclo). ${ }^{13} \mathrm{C} \mathrm{NMR}$ (101 MHz, $\mathrm{CDCl}_{3}, 25{ }^{\circ} \mathrm{C}$, TMS): $\delta=193.3,164.2,139.9,133.8$, $130.9,130.8,129.5,129.3,129.1,127.6,114.0,113.9,55.6,41.9$, 22.7, 15.1. IR (neat): $v$ 3026, 2929, 1735, 1657, 1600, 1570, $1510,1450,1427,1383,1308,1233,1170,1149,1085,1064$, 1024, 980, 888, 856, 819, $762 \mathrm{~cm}^{-1}$. HRMS (EI): $\mathrm{m} / \mathrm{z}$ calcd for $\mathrm{C}_{17} \mathrm{H}_{17} \mathrm{O}_{4} \mathrm{~S}[\mathrm{M}+\mathrm{H}]^{+}: 317.0848$, found: 317.0844 .

4.6. (4-Chlorophenyl)((1S,2R)-2-(phenylsulfonyl)cyclopropyl)methanone (4da)

Light yellow solid (56 mg, 88\% yield). mp. 122.8-123.6 ${ }^{\circ} \mathrm{C}$. ${ }^{1} \mathrm{H}$ NMR (400 MHz, $\mathrm{CDCl}_{3}, 25^{\circ} \mathrm{C}, \mathrm{TMS}$ ): $\delta=7.93$ (ddd, $J=8.8$, $6.2,5.1 \mathrm{~Hz}, 4 \mathrm{H}, \mathrm{ArH}), 7.72-7.61(\mathrm{~m}, 1 \mathrm{H}, \mathrm{ArH}), 7.57(\mathrm{dd}, J=$ 17.0, 9.6 Hz, 2H, ArH), $7.51-7.41$ (m, 2H, ArH), $3.54-3.41$ (m, 1H, CH-cyclo), 3.17 (ddd, $J=8.5,5.9,4.1 \mathrm{~Hz}, 1 \mathrm{H}, \mathrm{CH}-$ cyclo), 1.85 (ddd, $J=9.2,5.8,4.8 \mathrm{~Hz}, 1 \mathrm{H}, \mathrm{CH}_{2}$-cyclo), 1.74 (ddd, $J=8.5,5.7,4.9 \mathrm{~Hz}, 1 \mathrm{H}, \mathrm{CH}_{2}$-cyclo). ${ }^{13} \mathrm{C} \mathrm{NMR}(101 \mathrm{MHz}$, $\left.\mathrm{CDCl}_{3}, 25{ }^{\circ} \mathrm{C}, \mathrm{TMS}\right): \delta=194.0,140.5,139.7,134.7,134.0$, $129.8,129.5,129.2,127.7,42.3,22.9,15.4$. IR (neat): $v 3095$, 3039, 1676, 1584, 1483, 1446, 1402, 1374, 1310, 1287, 1258, 1212, 1148, 1083, 1022, 981, 915, 889, 866, 779, $754 \mathrm{~cm}^{-1}$. HRMS (EI): $\mathrm{m} / \mathrm{z}$ calcd for $\mathrm{C}_{16} \mathrm{H}_{14} \mathrm{ClO}_{3} \mathrm{~S}[\mathrm{M}+\mathrm{H}]^{+}$: 321.0352 , found: 321.0350 .

\section{7. (4-Bromophenyl)((1S,2R)-2-(phenylsulfonyl)- cyclopropyl)methanone (4ea)}

White solid (43 mg, 60\% yield). mp. 117.4-118.2 ${ }^{\circ} \mathrm{C} .{ }^{1} \mathrm{H}$ NMR (400 MHz, $\left.\mathrm{CDCl}_{3}, 25^{\circ} \mathrm{C}, \mathrm{TMS}\right): \delta=7.96-7.90$ (m, 2H, $\left.\mathrm{ArH}\right)$, $7.84(\mathrm{~d}, J=8.6 \mathrm{~Hz}, 2 \mathrm{H}, \mathrm{ArH}), 7.71-7.55(\mathrm{~m}, 5 \mathrm{H}, \mathrm{ArH}), 3.45$ (ddd, $J=9.5,5.8,4.1 \mathrm{~Hz}, 1 \mathrm{H}, \mathrm{CH}$-cyclo), 3.17 (ddd, $J=8.5,5.9$, $4.1 \mathrm{~Hz}, 1 \mathrm{H}, \mathrm{CH}$-cyclo), $1.90-1.81\left(\mathrm{~m}, 1 \mathrm{H}, \mathrm{CH}_{2}\right.$-cyclo), $1.78-$ $1.70\left(\mathrm{~m}, 1 \mathrm{H}, \mathrm{CH}_{2}\right.$-cyclo). ${ }^{13} \mathrm{C}$ NMR $\left(101 \mathrm{MHz}, \mathrm{CDCl}_{3}, 25{ }^{\circ} \mathrm{C}\right.$, TMS): $\delta=194.2,139.7,135.1,133.9,132.2,129.9,129.5,129.3$, 127.7, 42.3, 22.9, 15.4. IR (neat): $v$ 3029, 2924, 1734, 1671, $1583,1481,1447,1422,1402,1382,1308,1226,1175,1148$, $1069,1035,1009,985,921,857,826,750 \mathrm{~cm}^{-1}$. HRMS (EI): m/z calcd for $\mathrm{C}_{16} \mathrm{H}_{14} \mathrm{BrO}_{3} \mathrm{~S}[\mathrm{M}+\mathrm{H}]^{+}: 364.9847$, found: 364.9841 .

\section{8. (2-Bromophenyl)(( 1S,2R)-2-(phenylsulfonyl)- cyclopropyl)methanone (4fa)}

Yellowish oil (35 mg, 48\% yield). ${ }^{1} \mathrm{H}$ NMR (400 MHz, $\left.\mathrm{CDCl}_{3}, 25{ }^{\circ} \mathrm{C}, \mathrm{TMS}\right): \delta=7.93(\mathrm{~d}, J=7.3 \mathrm{~Hz}, 2 \mathrm{H}, \mathrm{ArH}), 7.62(\mathrm{dq}$, $J=30.7,7.6 \mathrm{~Hz}, 4 \mathrm{H}, \mathrm{ArH}), 7.45-7.30(\mathrm{~m}, 3 \mathrm{H}, \mathrm{ArH}), 3.39-$ 3.30 (m, 1H, CH-cyclo), 3.26 (ddd, $J=8.8,6.0,4.2 \mathrm{~Hz}, 1 \mathrm{H}, \mathrm{CH}-$ cyclo), $1.94-1.84\left(\mathrm{~m}, 1 \mathrm{H}, \mathrm{CH}_{2}\right.$-cyclo), $1.78(\mathrm{dt}, J=8.7,5.2 \mathrm{~Hz}$, $1 \mathrm{H}, \mathrm{CH}_{2}$-cyclo). ${ }^{13} \mathrm{C}$ NMR (101 MHz, $\left.\mathrm{CDCl}_{3}, 25{ }^{\circ} \mathrm{C}, \mathrm{TMS}\right): \delta=$ $198.7,140.2,139.7,133.9,133.9,132.6,129.5,127.8,127.6$, 119.4, 43.4, 26.9, 16.7. IR (neat): v 3062, 2923, 1690, 1586, 1468, 1445, 1430, 1379, 1315, 1287, 1213, 1188, 1152, 1087, 1069, 1026, 985, 952, 917, 861, $748 \mathrm{~cm}^{-1}$. HRMS (EI): $\mathrm{m} / \mathrm{z}$ calcd for $\mathrm{C}_{16} \mathrm{H}_{14} \mathrm{BrO}_{3} \mathrm{~S}[\mathrm{M}+\mathrm{H}]^{+}$: 364.9847 , found: 364.9833 .

\section{9. (4-Biphenyl)(( 1S,2R)-2-(phenylsulfonyl)cyclopropyl)- methanone (4ga)}

Light yellow solid (24 mg, 33\% yield). mp. 176.5-178.1 ${ }^{\circ} \mathrm{C}$. ${ }^{1} \mathrm{H}$ NMR (400 MHz, $\mathrm{CDCl}_{3}, 25{ }^{\circ} \mathrm{C}$, TMS): $\delta=8.06(\mathrm{~d}, J=8.4$ $\mathrm{Hz}, 2 \mathrm{H}, \mathrm{ArH}), 7.95$ (d, J = 7.5 Hz, 2H, ArH), $7.74-7.69$ (m, 2H, 
ArH), 7.65 (dd, $J=8.7,7.4 \mathrm{~Hz}, 3 \mathrm{H}, \mathrm{ArH}), 7.57$ (dd, $J=15.1$, $7.7 \mathrm{~Hz}, 2 \mathrm{H}, \mathrm{ArH}), 7.49$ (t, $J=7.4 \mathrm{~Hz}, 2 \mathrm{H}, \mathrm{ArH}), 7.42(\mathrm{t}, J=7.3$ $\mathrm{Hz}, 1 \mathrm{H}, \mathrm{ArH}), 3.56$ (ddd, $J=9.6,5.7,4.2 \mathrm{~Hz}, 1 \mathrm{H}, \mathrm{CH}$-cyclo), 3.20 (ddd, $J=8.6,5.8,4.2 \mathrm{~Hz}, 1 \mathrm{H}, \mathrm{CH}$-cyclo), 1.88 (dt, $J=9.3$, $5.3 \mathrm{~Hz}, 1 \mathrm{H}, \mathrm{CH}_{2}$-cyclo), $1.80-1.72\left(\mathrm{~m}, 1 \mathrm{H}, \mathrm{CH}_{2}\right.$-cyclo). ${ }^{13} \mathrm{C}$ NMR (101 MHz, $\left.\mathrm{CDCl}_{3}, 25{ }^{\circ} \mathrm{C}, \mathrm{TMS}\right): \delta=194.7,146.6,139.9$, $139.6,135.1,133.9,129.5,129.1,129.0,128.5,127.7,127.5$, $127.3,42.2,23.1,15.4$. IR (neat): $v$ 3027, 2923, 1733, 1663, $1601,1559,1516,1481,1448,1406,1381,1307,1267,1227$, 1207, 1183, 1150, 1085, 1067, 1028, 982, 920, 863, 839, $745 \mathrm{~cm}^{-}$ ${ }^{1}$. HRMS (EI): $\mathrm{m} / \mathrm{z}$ calcd for $\mathrm{C}_{22} \mathrm{H}_{19} \mathrm{O}_{3} \mathrm{~S}[\mathrm{M}+\mathrm{H}]^{+}$: 363.1055 , found: 363.1051 .

\subsection{0. (Furan-2-yl)(( 1S,2R)-2-(phenylsulfonyl)cyclopropyl)- methanone (4ha)}

White solid (43 mg, 78\% yield). mp. 140.6-142.2 ${ }^{\circ} \mathrm{C} .{ }^{1} \mathrm{H}$ NMR (400 MHz, $\left.\mathrm{CDCl}_{3}, 25^{\circ} \mathrm{C}, \mathrm{TMS}\right): \delta=7.95-7.91(\mathrm{~m}, 2 \mathrm{H}, \mathrm{ArH})$, $7.70-7.62(\mathrm{~m}, 2 \mathrm{H}, \mathrm{ArH}), 7.57(\mathrm{t}, J=7.6 \mathrm{~Hz}, 2 \mathrm{H}, \mathrm{ArH}), 7.31(\mathrm{t}$, $J=4.3 \mathrm{~Hz}, 1 \mathrm{H}, \mathrm{ArH}), 6.59(\mathrm{dd}, J=3.6,1.6 \mathrm{~Hz}, 1 \mathrm{H}, \mathrm{ArH}), 3.43$ (ddd, $J=9.6,5.7,4.2 \mathrm{~Hz}, 1 \mathrm{H}, \mathrm{CH}$-cyclo), 3.15 (ddd, $J=8.6,5.9$, $4.1 \mathrm{~Hz}, 1 \mathrm{H}, \mathrm{CH}$-cyclo), $1.89-1.78\left(\mathrm{~m}, 1 \mathrm{H}, \mathrm{CH}_{2}\right.$-cyclo), $1.78-$ $1.66\left(\mathrm{~m}, 1 \mathrm{H}, \mathrm{CH}_{2}\right.$-cyclo). ${ }^{13} \mathrm{C} \mathrm{NMR}\left(101 \mathrm{MHz}, \mathrm{CDCl}_{3}, 25{ }^{\circ} \mathrm{C}\right.$, TMS): $\delta=183.4,152.2,147.6,139.8,133.9,129.4,127.7,118.6$, $112.8,41.8,23.2,14.9$. IR (neat): $v$ 3039, 2924, 1735, 1664, 1568, 1466, 1403, 1381, 1308, 1286, 1234, 1196, 1153, 1084, 1039, 1015, 992, 920, 879, 855, 794, $755 \mathrm{~cm}^{-1}$. HRMS (EI): m/z calcd for $\mathrm{C}_{14} \mathrm{H}_{13} \mathrm{O}_{4} \mathrm{~S}[\mathrm{M}+\mathrm{H}]^{+}: 277.0535$, found: 277.0532 .

\subsection{1. ((1S,2R)-2-(Phenylsulfonyl)cyclopropyl)(thiophen-2- yl)methanone (4ia)}

Yellow solid (43 mg, 78\% yield). mp. 120.1-121.8 ${ }^{\circ} \mathrm{C} .{ }^{1} \mathrm{H}$ NMR (400 MHz, $\left.\mathrm{CDCl}_{3}, 25^{\circ} \mathrm{C}, \mathrm{TMS}\right): \delta=7.95-7.90(\mathrm{~m}, 2 \mathrm{H}$, ArH), $7.88(\mathrm{dd}, J=3.8,0.8 \mathrm{~Hz}, 1 \mathrm{H}, \mathrm{ArH}), 7.72(\mathrm{dd}, J=4.9,0.8$ $\mathrm{Hz}, 1 \mathrm{H}, \mathrm{ArH}), 7.66(\mathrm{t}, J=7.4 \mathrm{~Hz}, 1 \mathrm{H}, \mathrm{ArH}), 7.57(\mathrm{t}, J=7.6 \mathrm{~Hz}$, 2H, ArH), $7.18(\mathrm{dd}, J=4.7,4.0 \mathrm{~Hz}, 1 \mathrm{H}, \mathrm{ArH}), 3.43-3.33(\mathrm{~m}$, $1 \mathrm{H}, \mathrm{CH}$-cyclo), 3.18 (ddd, $J=8.6,5.9,4.1 \mathrm{~Hz}, 1 \mathrm{H}, \mathrm{CH}$-cyclo), 1.84 (ddd, $J=9.2,5.8,5.0 \mathrm{~Hz}, 1 \mathrm{H}, \mathrm{CH}_{2}$-cyclo), 1.74 (dt, $J=8.6$, $5.3 \mathrm{~Hz}, 1 \mathrm{H}, \mathrm{CH}_{2}$-cyclo). ${ }^{13} \mathrm{C} \mathrm{NMR}\left(101 \mathrm{MHz}, \mathrm{CDCl}_{3}, 25{ }^{\circ} \mathrm{C}\right.$, TMS): $\delta=187.4,143.4,139.8,135.2,133.9,133.2,129.5,128.6$, 127.7, 41.9, 23.9, 15.1. IR (neat): $v$ 3022, 2924, 1731, 1652, $1515,1447,1413,1381,1353,1305,1246,1223,1194,1147$, 1086, 1065, 1022, 957, 920, 864, 843, $727 \mathrm{~cm}^{-1}$. HRMS (EI): m/z calcd for $\mathrm{C}_{14} \mathrm{H}_{13} \mathrm{O}_{3} \mathrm{~S}_{2}[\mathrm{M}+\mathrm{H}]^{+}:$293.0306, found: 293.0301 .

\subsection{2. (Naphthalen-4-yl)((1S,2R)-2-(phenylsulfonyl)- cyclopropyl)methanone (4ja)}

Yellow solid (59 mg, $72 \%$ yield). mp. $133.6-134.7{ }^{\circ} \mathrm{C} .{ }^{1} \mathrm{H}$ NMR (400 MHz, $\mathrm{CDCl}_{3}, 25^{\circ} \mathrm{C}$, TMS): $\delta=8.42(\mathrm{~d}, J=8.3 \mathrm{~Hz}$, 1H, ArH), $7.94(\mathrm{~d}, J=8.2 \mathrm{~Hz}, 1 \mathrm{H}, \mathrm{ArH}), 7.92-7.85(\mathrm{~m}, 3 \mathrm{H}$, ArH), 7.79 (dd, $J=9.3,7.9 \mathrm{~Hz}, 1 \mathrm{H}, \mathrm{ArH}), 7.60(\mathrm{t}, J=7.4 \mathrm{~Hz}$, 1H, ArH), 7.47 (dddd, $J=15.5,11.5,8.9,5.9 \mathrm{~Hz}, 5 \mathrm{H}, \mathrm{ArH}), 3.45$ -3.35 (m, 1H, CH-cyclo), 3.21 (ddd, $J=8.5,5.9,4.1 \mathrm{~Hz}, 1 \mathrm{H}$, $\mathrm{CH}$-cyclo), 1.80 (ddt, $J=10.5,8.5,4.7 \mathrm{~Hz}, 2 \mathrm{H}, \mathrm{CH}_{2}$-cyclo). ${ }^{13} \mathrm{C}$ NMR (101 MHz, $\mathrm{CDCl}_{3}, 25{ }^{\circ} \mathrm{C}$, TMS): $\delta=198.27,139.87$, 134.94, 133.94, 133.86, 133.66, 129.96, 129.53, 129.04, 128.58, $128.28,127.75,126.72,125.50,124.49,42.80,26.28,15.83$. IR (neat): $v$ 3029, 2924, 1729, 1663, 1585, 1506, 1447, 1400, 1372, 1308, 1278, 1231, 1149, 1101, 1069, 1025, 960, 920, 889, 864, 809, 778, $733 \mathrm{~cm}^{-1}$. HRMS (EI): $\mathrm{m} / \mathrm{z}$ calcd for $\mathrm{C}_{20} \mathrm{H}_{17} \mathrm{O}_{3} \mathrm{~S}$ $[\mathrm{M}+\mathrm{H}]^{+}:$337.0898, found: 337.0892 .

\subsection{3. (1,9-Dihydropyren-5-yl)((1S,2R)-2-(phenylsulfonyl)- cyclopropyl)methanone (4ka)}

Yellow solid (59 mg, 72\% yield). mp. $162.8-163.2{ }^{\circ} \mathrm{C} .{ }^{1} \mathrm{H}$ NMR (400 MHz, $\left.\mathrm{CDCl}_{3}, 25^{\circ} \mathrm{C}, \mathrm{TMS}\right): \delta=8.77(\mathrm{~d}, J=9.4 \mathrm{~Hz}$,
$1 \mathrm{H}, \mathrm{ArH}), 8.35$ (d, $J=8.0 \mathrm{~Hz}, 1 \mathrm{H}, \mathrm{ArH}), 8.21(\mathrm{~d}, J=7.7 \mathrm{~Hz}, 2 \mathrm{H}$, ArH), $8.18-8.07$ (m, 3H, ArH), $8.06-7.96$ (m, 4H, ArH), 7.69 - $7.62(\mathrm{~m}, 1 \mathrm{H}, \mathrm{ArH}), 7.58$ (t, $J=7.5 \mathrm{~Hz}, 2 \mathrm{H}, \mathrm{ArH}), 3.67-3.60$ (m, 1H, CH-cyclo), 3.38 (ddd, $J=8.1,6.2,4.1 \mathrm{~Hz}, 1 \mathrm{H}, \mathrm{CH}-$ cyclo), $2.01-1.92$ (m, 2H, $\mathrm{CH}_{2}$-cyclo). ${ }^{13} \mathrm{C}$ NMR $(101 \mathrm{MHz}$, $\left.\mathrm{CDCl}_{3}, 25{ }^{\circ} \mathrm{C}, \mathrm{TMS}\right): \delta=198.4,134.0,134.4,133.9,131.1$, $131.0,130.4,130.03,130.00,129.5,127.8,127.1,127.0,126.6$, $126.5,126.4,124.8,124.4,124.1,43.0,26.7,16.0$. IR (neat): $v$ 3043, 2957, 2922, 1735, 1659, 1587, 1537, 1502, 1446, 1381, 1311, 1259, 1214, 1184, 1145, 1116, 1080, 1021, 982, 959, 918, 889, 844, 782, 765, $747 \mathrm{~cm}^{-1}$. HRMS (EI): $\mathrm{m} / \mathrm{z}$ calcd for $\mathrm{C}_{26} \mathrm{H}_{21} \mathrm{O}_{3} \mathrm{~S}[\mathrm{M}+\mathrm{H}]^{+}:$413.1211, found: 413.1209.

\subsection{Phenyl((1S,2R)-2-tosylcyclopropyl)methanone (4ab)}

Light yellow solid (30 mg, 50\% yield). mp. 117.4-118.6 ${ }^{\circ} \mathrm{C}$. ${ }^{1} \mathrm{H}$ NMR (400 MHz, $\mathrm{CDCl}_{3}, 25{ }^{\circ} \mathrm{C}$, TMS): $\delta=8.04-7.95(\mathrm{~m}$, $2 \mathrm{H}, \mathrm{ArH}), 7.79(\mathrm{t}, J=9.8 \mathrm{~Hz}, 2 \mathrm{H}, \mathrm{ArH}), 7.61(\mathrm{t}, J=7.4 \mathrm{~Hz}, 1 \mathrm{H}$, ArH), $7.48(\mathrm{dd}, J=17.0,9.5 \mathrm{~Hz}, 2 \mathrm{H}, \mathrm{ArH}), 7.36(\mathrm{~d}, J=8.0 \mathrm{~Hz}$, 2H, ArH), $3.55-3.45$ (m, 1H, CH-cyclo), 3.16 (ddd, $J=8.6,5.8$, $4.2 \mathrm{~Hz}, 1 \mathrm{H}, \mathrm{CH}$-cyclo), 2.45 (s, 3H, $\mathrm{CH}_{3}$ ), 1.84 (ddd, $J=9.3,5.7$, $4.8 \mathrm{~Hz}, 1 \mathrm{H}, \mathrm{CH}_{2}$-cyclo), $1.76-1.68\left(\mathrm{~m}, 1 \mathrm{H}, \mathrm{CH}_{2}\right.$-cyclo). ${ }^{13} \mathrm{C}$ NMR (101 MHz, $\left.\mathrm{CDCl}_{3}, 25^{\circ} \mathrm{C}, \mathrm{TMS}\right): \delta=195.3,144.9,136.9$, $136.5,133.8,130.1,128.8,128.4,127.7,42.4,23.1,21.6,15.3$. IR (neat): $v$ 3046, 2923, 1734, 1682, 1593, 1456, 1383, 1317 , 1268, 1217, 1145, 1086, 1025, 986, 917, 883, 815, 798, $746 \mathrm{~cm}^{-1}$. HRMS (EI): $\mathrm{m} / \mathrm{z}$ calcd for $\mathrm{C}_{17} \mathrm{H}_{17} \mathrm{O}_{3} \mathrm{~S}[\mathrm{M}+\mathrm{H}]^{+}: 301.0898$, found: 301.0879 .

\subsection{5. ((1S,2R)-2-(4-Methoxyphenylsulfonyl)cyclopropyl) (phenyl)-methanone (4ac)}

Yellow solid (42 mg, 66\% yield). mp. $122.3-123.4{ }^{\circ} \mathrm{C} .{ }^{1} \mathrm{H}$ NMR (400 MHz, $\left.\mathrm{CDCl}_{3}, 25{ }^{\circ} \mathrm{C}, \mathrm{TMS}\right): \delta=7.99(\mathrm{~d}, J=7.4 \mathrm{~Hz}$, $2 \mathrm{H}, \mathrm{ArH}), 7.85(\mathrm{~d}, J=8.9 \mathrm{~Hz}, 2 \mathrm{H}, \mathrm{ArH}), 7.61(\mathrm{t}, J=7.4 \mathrm{~Hz}, 1 \mathrm{H}$, ArH), 7.49 (t, $J=7.7 \mathrm{~Hz}, 2 \mathrm{H}, \mathrm{ArH}), 7.03(\mathrm{t}, J=7.4 \mathrm{~Hz}, 2 \mathrm{H}$, ArH), 3.88 (s, 3H, $\mathrm{OCH}_{3}$ ), 3.49 (ddd, $J=9.5,5.7,4.2 \mathrm{~Hz}, 1 \mathrm{H}$, CH-cyclo), 3.15 (ddd, $J=8.6,5.8,4.2 \mathrm{~Hz}, 1 \mathrm{H}, \mathrm{CH}$-cyclo), 1.87 1.79 (m, 1H, $\mathrm{CH}_{2}$-cyclo), $1.75-1.69$ (m, $1 \mathrm{H}, \mathrm{CH}_{2}$-cyclo). ${ }^{13} \mathrm{C}$ NMR (101 MHz, $\left.\mathrm{CDCl}_{3}, 25^{\circ} \mathrm{C}, \mathrm{TMS}\right): \delta=195.3,163.9,136.5$, $133.8,131.4,129.9,128.8,128.4,114.7,55.7,42.6,23.1,15.4$. IR (neat): $v$ 3044, 2936, 1734, 1672, 1594, 1497, 1387, 1297 , 1262, 1222, 1146, 1089, 1024, 987, 858, 801, $741 \mathrm{~cm}^{-1}$. HRMS (EI): $\mathrm{m} / \mathrm{z}$ calcd for $\mathrm{C}_{16} \mathrm{H}_{13} \mathrm{O}_{3} \mathrm{~S}[\mathrm{M}+\mathrm{H}]^{+}:$285.0585, found: 285.0577 .

\subsection{6. ((1S,2R)-2-(4-Chlorophenylsulfonyl)cyclopropyl)(phenyl)- methanone (4ad)}

Light yellow solid (33 mg, 51\% yield). mp. 127.8-129.1 ${ }^{\circ} \mathrm{C}$. ${ }^{1} \mathrm{H}$ NMR (400 MHz, $\mathrm{CDCl}_{3}, 25{ }^{\circ} \mathrm{C}$, TMS): $\delta=7.99(\mathrm{~d}, J=7.5$ $\mathrm{Hz}, 2 \mathrm{H}, \mathrm{ArH}), 7.87$ (d, $J=8.5 \mathrm{~Hz}, 2 \mathrm{H}, \mathrm{ArH}), 7.63(\mathrm{t}, J=7.6 \mathrm{~Hz}$, $1 \mathrm{H}, \mathrm{ArH}), 7.57-7.47$ (m, 4H, ArH), $3.56-3.47(\mathrm{~m}, 1 \mathrm{H}, \mathrm{CH}-$ cyclo), $3.22-3.13\left(\mathrm{~m}, 1 \mathrm{H}, \mathrm{CH}\right.$-cyclo), $1.88-1.80\left(\mathrm{~m}, 1 \mathrm{H}, \mathrm{CH}_{2^{-}}\right.$ cyclo), 1.73 (dt, $J=8.6,5.3 \mathrm{~Hz}, 1 \mathrm{H}, \mathrm{CH}_{2}$-cyclo). ${ }^{13} \mathrm{C}$ NMR $(101$ $\mathrm{MHz}, \mathrm{CDCl}_{3}, 25{ }^{\circ} \mathrm{C}$, TMS): $\delta=194.9,140.7,138.3,136.3$, 133.9, 129.8, 129.2, 128.9, 128.4, 42.0, 23.1, 15.4. IR (neat): $v$ 3047, 2923, 1732, 1671, 1578, 1473, 1449, 1389, 1308, 1270, 1222, 1151, 1086, 1018, 984, 919, 887, 856, 830, 794, 760, 731 $\mathrm{cm}^{-1}$. HRMS (EI): $\mathrm{m} / \mathrm{z}$ calcd for $\mathrm{C}_{16} \mathrm{H}_{14} \mathrm{ClO}_{3} \mathrm{~S}[\mathrm{M}+\mathrm{H}]^{+}: 321.0352$, found: 321.0350 .

\subsection{7. ((1S,2R)-2-(2-Thienylsulfonyl)cyclopropyl)(phenyl)- methanone (4ae)}

Yellow solid (40 mg, 68\% yield). mp. 113.4-114.2 ${ }^{\circ} \mathrm{C} .{ }^{1} \mathrm{H}$ NMR (400 MHz, $\left.\mathrm{CDCl}_{3}, 25{ }^{\circ} \mathrm{C}, \mathrm{TMS}\right): \delta=8.04-7.98(\mathrm{~m}, 2 \mathrm{H}$, ArH), 7.73 (ddd, $J=6.1,4.3,1.1 \mathrm{~Hz}, 2 \mathrm{H}, \mathrm{ArH}), 7.62(\mathrm{t}, J=7.4$ $\mathrm{Hz}, 1 \mathrm{H}, \mathrm{ArH}$ ), 7.50 (t, $J=7.7 \mathrm{~Hz}, 2 \mathrm{H}, \mathrm{ArH}), 7.16$ (dd, $J=4.8$, 
$3.9 \mathrm{~Hz}, 1 \mathrm{H}, \mathrm{ArH}), 3.54$ (ddd, $J=9.6,5.8,4.1 \mathrm{~Hz}, 1 \mathrm{H}, \mathrm{CH}$-cyclo), 3.30 (ddd, $J=8.6,5.8,4.1 \mathrm{~Hz}, 1 \mathrm{H}, \mathrm{CH}$-cyclo), $1.95-1.86(\mathrm{~m}$, $1 \mathrm{H}, \mathrm{CH}_{2}$-cyclo), $1.82-1.75$ (m, $1 \mathrm{H}, \mathrm{CH}_{2}$-cyclo). ${ }^{13} \mathrm{C} \mathrm{NMR}(101$ $\left.\mathrm{MHz}, \mathrm{CDCl}_{3}, 25^{\circ} \mathrm{C}, \mathrm{TMS}\right): \delta=195.0,140.5,136.3,134.2,133.9$, $128.9,128.5,128.0,43.4,23.7,15.8$. IR (neat): $v$ 3088, 3048, $1733,1676,1595,1449,1401,1380,1314,1265,1225,1142$, 1097, 1068, 1016, 984, 863, $736 \mathrm{~cm}^{-1}$. HRMS (EI): m/z calcd for $\mathrm{C}_{14} \mathrm{H}_{13} \mathrm{O}_{3} \mathrm{~S}_{2}[\mathrm{M}+\mathrm{H}]^{+}:$293.0306, found: 293.0298 .

\subsection{8. p-Tolyl((1S,2R)-2-tosylcyclopropyl)methanone (4bb)}

Light yellow solid (32 mg, 51\% yield). mp. 143.7-145.2 ${ }^{\circ} \mathrm{C}$. ${ }^{1} \mathrm{H}$ NMR $\left(400 \mathrm{MHz}, \mathrm{CDCl}_{3}, 25{ }^{\circ} \mathrm{C}\right.$, TMS): $\delta=7.89$ (d, $J=8.2$ $\mathrm{Hz}, 2 \mathrm{H}, \mathrm{ArH}), 7.80$ (d, $J=8.3 \mathrm{~Hz}, 2 \mathrm{H}, \mathrm{ArH}), 7.35$ (d, $J=8.0 \mathrm{~Hz}$, $2 \mathrm{H}, \mathrm{ArH}), 7.28(\mathrm{~d}, J=8.0 \mathrm{~Hz}, 2 \mathrm{H}, \operatorname{ArH}), 3.51-3.44(\mathrm{~m}, 1 \mathrm{H}$, CH-cyclo), 3.14 (ddd, $J=8.5,5.8,4.1 \mathrm{~Hz}, 1 \mathrm{H}, \mathrm{CH}$-cyclo), 2.44 (s, $\left.3 \mathrm{H}, \mathrm{CH}_{3}\right), 2.43\left(\mathrm{~s}, 3 \mathrm{H}, \mathrm{CH}_{3}\right), 1.81$ (ddd, $J=9.2,5.8,4.7 \mathrm{~Hz}$, $1 \mathrm{H}, \mathrm{CH}_{2}$-cyclo), $1.73-1.69$ (m, $1 \mathrm{H}, \mathrm{CH}_{2}$-cyclo). ${ }^{13} \mathrm{C} \mathrm{NMR}(101$ $\left.\mathrm{MHz}, \mathrm{CDCl}_{3}, 25{ }^{\circ} \mathrm{C}, \mathrm{TMS}\right): \delta=194.7,144.9,144.9,137.0$, 134.0, 130.1, 129.5, 128.6, 127.7, 42.2, 22.9, 21.7, 21.6, 15.2. IR (neat): $v$ 3028, 2923, 1732, 1661, 1602, 1491, 1455, 1419, 1383, 1305, 1271, 1232, 1210, 1178, 1145, 1085, 1064, 1032, 986, 919, 860, 818, 759, $716 \mathrm{~cm}^{-1}$. HRMS (EI): $\mathrm{m} / \mathrm{z}$ calcd for $\mathrm{C}_{18} \mathrm{H}_{19} \mathrm{O}_{3} \mathrm{~S}$ $[\mathrm{M}+\mathrm{H}]^{+}: 315.1055$, found: 315.1049 .

\subsection{9. ((1S,2R)-2-(4-Chlorophenylsulfonyl)cyclopropyl)(p- tolyl)methanone $(4 \mathrm{bd})$}

Light yellow solid (22 mg, 33\% yield). mp. 156.2-157.1 ${ }^{\circ} \mathrm{C}$. ${ }^{1} \mathrm{H}$ NMR $\left(400 \mathrm{MHz}, \mathrm{CDCl}_{3}, 25{ }^{\circ} \mathrm{C}\right.$, TMS $): \delta=7.96-7.80(\mathrm{~m}$, $4 \mathrm{H}, \mathrm{ArH}), 7.59-7.51(\mathrm{~m}, 2 \mathrm{H}, \mathrm{ArH}), 7.29(\mathrm{t}, J=6.9 \mathrm{~Hz}, 2 \mathrm{H}$, ArH), 3.49 (ddd, $J=9.6,5.8,4.1 \mathrm{~Hz}, 1 \mathrm{H}, \mathrm{CH}$-cyclo), 3.15 (ddd, $J$ $=8.5,5.8,4.1 \mathrm{~Hz}, 1 \mathrm{H}, \mathrm{CH}$-cyclo), $2.44\left(\mathrm{~s}, 3 \mathrm{H}, \mathrm{CH}_{3}\right), 1.82$ (ddd, $J$ $=9.3,5.7,4.8 \mathrm{~Hz}, 1 \mathrm{H}, \mathrm{CH}_{2}$-cyclo), 1.72 (ddd, $J=8.5,5.8,4.8$ $\mathrm{Hz}, 1 \mathrm{H}, \mathrm{CH}_{2}$-cyclo). ${ }^{13} \mathrm{C} \mathrm{NMR}\left(101 \mathrm{MHz}, \mathrm{CDCl}_{3}, 25{ }^{\circ} \mathrm{C}, \mathrm{TMS}\right)$ : $\delta=194.4,145.0,140.7,138.4,133.9,129.8,129.6,129.2,128.6$, 41.9, 22.9, 21.7, 15.3. IR (neat): $v$ 3039, 2920, 1733, 1663, 1604, $1574,1470,1423,1386,1322,1269,1232,1205,1177,1147$, 1086, 1035, 1012, 990, 861, 827, 761, $721 \mathrm{~cm}^{-1}$. HRMS (EI): $\mathrm{m} / \mathrm{z}$ calcd for $\mathrm{C}_{17} \mathrm{H}_{16} \mathrm{ClO}_{3} \mathrm{~S}[\mathrm{M}+\mathrm{H}]^{+}$: 335.0509, found: 335.0504 .

The procedure for crystal growth: Compoud $\mathbf{4 b d}$ was soulble in mixtures of chloroform and petroleum ether, crystal could be prepared by the slow volatilization of solvents at room temperature.

\subsection{0. (4-Chlorophenyl)(( 1S,2R)-2-tosylcyclopropyl)methanone (4db)}

Light yellow solid (42 mg, 63\% yield). mp. 130.4-132.3 ${ }^{\circ} \mathrm{C}$. ${ }^{1} \mathrm{H}$ NMR (400 MHz, $\left.\mathrm{CDCl}_{3}, 25^{\circ} \mathrm{C}, \mathrm{TMS}\right): \delta=7.94$ (dd, $J=11.4$, $9.6 \mathrm{~Hz}, 2 \mathrm{H}, \mathrm{ArH}), 7.80$ (d, $J=8.3 \mathrm{~Hz}, 2 \mathrm{H}, \mathrm{ArH}), 7.49-7.44(\mathrm{~m}$, 2H, ArH), 7.36 (d, $J=8.0 \mathrm{~Hz}, 2 \mathrm{H}, \mathrm{ArH}), 3.44$ (ddd, $J=9.5,5.8$, $4.1 \mathrm{~Hz}, 1 \mathrm{H}, \mathrm{CH}$-cyclo), 3.14 (ddd, $J=8.5,5.9,4.1 \mathrm{~Hz}, 1 \mathrm{H}, \mathrm{CH}-$ cyclo), 2.45 (s, $3 \mathrm{H}, \mathrm{CH}_{3}$ ), 1.83 (ddd, $J=9.2,5.8,4.8 \mathrm{~Hz}, 1 \mathrm{H}$, $\mathrm{CH}_{2}$-cyclo), $1.79-1.67\left(\mathrm{~m}, 1 \mathrm{H}, \mathrm{CH}_{2}\right.$-cyclo). ${ }^{13} \mathrm{C}$ NMR $(101$ $\left.\mathrm{MHz}, \mathrm{CDCl}_{3}, 25{ }^{\circ} \mathrm{C}, \mathrm{TMS}\right): \delta=194.1,145.0,140.4,136.8$, $134.8,130.1,129.8,129.2,127.7,42.5,22.9,21.6,15.4$. IR (neat): v 3042, 2922, 1736, 1667, 1588, 1487, 1425, 1404, 1384, 1318, 1289, 1266, 1174, 1147, 1116, 1089, 1066, 1034, 986, 925, $862,834,814,780,755 \mathrm{~cm}^{-1}$. HRMS (EI): $\mathrm{m} / \mathrm{z}$ calcd for $\mathrm{C}_{17} \mathrm{H}_{16} \mathrm{ClO}_{3} \mathrm{~S}[\mathrm{M}+\mathrm{H}]^{+}:$335.0509, found: 335.0501 .

\subsection{1. ((1S,2R)-2-(4-Chlorophenylsulfonyl)cyclopropyl)(4- chlorophenyl)methanone (4dd)}

Light yellow solid (30 mg, 43\% yield). mp. 160.4-161.5 ${ }^{\circ} \mathrm{C}$. ${ }^{1} \mathrm{H}$ NMR $\left(400 \mathrm{MHz}, \mathrm{CDCl}_{3}, 25{ }^{\circ} \mathrm{C}, \mathrm{TMS}\right): \delta=7.97-7.92(\mathrm{~m}$, $2 \mathrm{H}, \mathrm{ArH}), 7.88-7.84$ (m, 2H, ArH), $7.58-7.54(\mathrm{~m}, 2 \mathrm{H}, \mathrm{ArH})$, $7.50-7.46(\mathrm{~m}, 2 \mathrm{H}, \mathrm{ArH}), 3.46(\mathrm{ddd}, J=9.5,5.8,4.1 \mathrm{~Hz}, 1 \mathrm{H}$,
CH-cyclo), 3.16 (ddd, $J=8.5,5.9,4.1 \mathrm{~Hz}, 1 \mathrm{H}, \mathrm{CH}$-cyclo), 1.83 (ddd, $J=9.2,5.8,4.8 \mathrm{~Hz}, 1 \mathrm{H}, \mathrm{CH}_{2}$-cyclo), 1.73 (ddd, $J=8.5$, 5.7, $4.9 \mathrm{~Hz}, 1 \mathrm{H}, \mathrm{CH}_{2}$-cyclo). ${ }^{13} \mathrm{C} \mathrm{NMR}\left(101 \mathrm{MHz}, \mathrm{CDCl}_{3}, 25{ }^{\circ} \mathrm{C}\right.$, TMS): $\delta=193.8,140.8,140.6,138.2,134.6,129.9,129.8,129.2$, 129.2, 42.1, 22.9, 15.5. IR (neat): v 3090, 2924, 1730, 1670, 1585, 1476, 1426, 1400, 1308, 1276, 1221, 1178, 1146, 1087, 1036, 991, 865, 766, $751 \mathrm{~cm}^{-1}$. HRMS (EI): $\mathrm{m} / \mathrm{z}$ calcd for $\mathrm{C}_{16} \mathrm{H}_{13} \mathrm{Cl}_{2} \mathrm{O}_{3} \mathrm{~S}[\mathrm{M}+\mathrm{H}]^{+}$: 354.9962, found: 354.9951 .

\subsection{2. (Thiophen-2-yl)((1S,2R)-2-tosylcyclopropyl)methanone (4ib)}

Light yellow solid (31 mg, 51\% yield). mp. 126.3-127.6 ${ }^{\circ} \mathrm{C}$. ${ }^{1} \mathrm{H}$ NMR (400 MHz, $\mathrm{CDCl}_{3}, 25{ }^{\circ} \mathrm{C}$, TMS): $\delta=7.88$ (dd, $J=5.8$, $4.9 \mathrm{~Hz}, 1 \mathrm{H}, \mathrm{ArH}), 7.79(\mathrm{t}, J=7.9 \mathrm{~Hz}, 2 \mathrm{H}, \mathrm{ArH}), 7.74-7.71(\mathrm{~m}$, $1 \mathrm{H}, \mathrm{ArH}), 7.36(\mathrm{~d}, J=8.1 \mathrm{~Hz}, 2 \mathrm{H}, \mathrm{ArH}), 7.18(\mathrm{dd}, J=4.8,4.0$ $\mathrm{Hz}, 1 \mathrm{H}, \mathrm{ArH}$ ), $3.40-3.33$ (m, 1H, CH-cyclo), 3.16 (ddd, $J=8.7$, 5.9, $4.1 \mathrm{~Hz}, 1 \mathrm{H}, \mathrm{CH}$-cyclo), $2.44\left(\mathrm{~s}, 3 \mathrm{H}, \mathrm{CH}_{3}\right), 1.82$ (ddd, $J=9.2$, $5.8,4.9 \mathrm{~Hz}, 1 \mathrm{H}, \mathrm{CH}_{2}$-cyclo), $1.72\left(\mathrm{dt}, J=8.6,5.2 \mathrm{~Hz}, 1 \mathrm{H}, \mathrm{CH}_{2^{-}}\right.$ cyclo). ${ }^{13} \mathrm{C}$ NMR (101 MHz, $\mathrm{CDCl}_{3}, 25{ }^{\circ} \mathrm{C}$, TMS $): \delta=187.5$, 144.9, 143.5, 136.8, 135.1, 133.2, 130.1, 128.6, 127.7, 42.1, 23.9, 21.7, 15.1. IR (neat): $v$ 3022, 2923, 1724, 1645, 1594, 1515, 1454, 1416, 1382, 1293, 1247, 1222, 1189, 1142, 1109, 1084, $1065,1020,957,921,864,842,818,758 \mathrm{~cm}^{-1}$. HRMS (EI): m/z calcd for $\mathrm{C}_{15} \mathrm{H}_{15} \mathrm{O}_{3} \mathrm{~S}_{2}[\mathrm{M}+\mathrm{H}]^{+}: 307.0463$, found: 307.0461 .

\subsection{3. ((1S,2R)-2-(4-Chlorophenylsulfonyl)cyclopropyl)- (thiophen-2-yl)methanone (4id)}

Light yellow solid (13 mg, 20\% yield). mp. 170.4-171.8 ${ }^{\circ} \mathrm{C}$. ${ }^{1} \mathrm{H}$ NMR (400 MHz, $\mathrm{CDCl}_{3}, 25{ }^{\circ} \mathrm{C}$, TMS): $\delta=7.89$ (dd, $J=3.8$, $1.0 \mathrm{~Hz}, 1 \mathrm{H}, \mathrm{ArH}), 7.88-7.83(\mathrm{~m}, 2 \mathrm{H}, \mathrm{ArH}), 7.74(\mathrm{dd}, J=5.0$, $1.0 \mathrm{~Hz}, 1 \mathrm{H}, \mathrm{ArH}), 7.58-7.52(\mathrm{~m}, 2 \mathrm{H}, \mathrm{ArH}), 7.20(\mathrm{dd}, J=4.9$, $3.9 \mathrm{~Hz}, 1 \mathrm{H}, \mathrm{ArH}), 3.44-3.33$ (m, 1H, CH-cyclo), $3.21-3.12$ (m, $1 \mathrm{H}, \mathrm{CH}$-cyclo), 1.83 (ddd, $J=9.2,5.8,4.9 \mathrm{~Hz}, 1 \mathrm{H}, \mathrm{CH}_{2^{-}}$ cyclo), $1.78-1.71\left(\mathrm{~m}, 1 \mathrm{H}, \mathrm{CH}_{2}\right.$-cyclo). ${ }^{13} \mathrm{C} \mathrm{NMR}(101 \mathrm{MHz}$, $\left.\mathrm{CDCl}_{3}, 25{ }^{\circ} \mathrm{C}, \mathrm{TMS}\right): \delta=187.2,143.3,140.8,138.2,135.3$, 133.3, 129.9, 129.2, 128.6, 41.8, 23.9, 15.2. IR (neat): $v$ 3042, 2923, 1724, 1650, 1578, 1513, 1473, 1412, 1352, 1308, 1275, 1238, 1220, 1150, 1085, 1011, 957, 919, 861, 847, 831, 764, 740 $\mathrm{cm}^{-1}$. HRMS (EI): $\mathrm{m} / \mathrm{z}$ calcd for $\mathrm{C}_{14} \mathrm{H}_{12} \mathrm{ClO}_{3} \mathrm{~S}_{2}[\mathrm{M}+\mathrm{H}]^{+}$: 326.9916, found: 326.9911 .

\subsection{Ethyl 2-benzoyl-1-phenylsulfonylcyclopropanecarboxylate} (3aa)

Yellowish oil. ${ }^{1} \mathrm{H}$ NMR (400 MHz, $\left.\mathrm{CDCl}_{3}, 25^{\circ} \mathrm{C}, \mathrm{TMS}\right): \delta=$ $8.02-7.97$ (m, 2H, ArH), $7.97-7.92(\mathrm{~m}, 2 \mathrm{H}, \mathrm{ArH}), 7.71(\mathrm{t}, J=$ $7.5 \mathrm{~Hz}, 1 \mathrm{H}, \mathrm{ArH}), 7.63-7.56(\mathrm{~m}, 3 \mathrm{H}, \mathrm{ArH}), 7.45(\mathrm{t}, J=7.7 \mathrm{~Hz}$, $2 \mathrm{H}, \mathrm{ArH}), 4.02-3.93(\mathrm{~m}, 2 \mathrm{H}, \mathrm{COOEt}), 3.66(\mathrm{dd}, J=9.4,7.5 \mathrm{~Hz}$, $1 \mathrm{H}, \mathrm{CH}$-cyclo), 2.36 (dd, $J=7.4,5.4 \mathrm{~Hz}, 1 \mathrm{H}, \mathrm{CH}_{2}$-cyclo), 2.24 (dd, $J=9.5,5.3 \mathrm{~Hz}, 1 \mathrm{H}, \mathrm{CH}_{2}$-cyclo), $0.97(\mathrm{t}, J=7.1 \mathrm{~Hz}, 3 \mathrm{H}$, COOEt). ${ }^{13} \mathrm{C}$ NMR (101 MHz, $\left.\mathrm{CDCl}_{3}, 25{ }^{\circ} \mathrm{C}, \mathrm{TMS}\right): \delta=192.4$, $163.3,138.4,136.4,134.3,133.8,129.3,129.1,128.8,128.6$, 62.5, 53.3, 30.1, 18.4, 13.5. IR (neat): v 3065, 2985, 1736, 1681, 1598, 1582, 1449, 1372, 1310, 1228, 1202, 1161, 1138, 1081, 1017, 933, 871, 856, 827, 756, $728 \mathrm{~cm}^{-1}$. HRMS (EI): $\mathrm{m} / \mathrm{z}$ calcd for $\mathrm{C}_{19} \mathrm{H}_{19} \mathrm{O}_{5} \mathrm{~S}[\mathrm{M}+\mathrm{H}]^{+}: 359.0953$, found: 359.0952 .

\subsection{Ethyl 2-benzoyl-1-methylsulfonylcyclopropanecarboxylate (3af)}

Yellowish oil (32 mg, 53\% yield). ${ }^{1} \mathrm{H}$ NMR (400 MHz, $\left.\mathrm{CDCl}_{3}, 25^{\circ} \mathrm{C}, \mathrm{TMS}\right): \delta=8.13-7.95(\mathrm{~m}, 2 \mathrm{H}, \mathrm{ArH}), 7.63(\mathrm{t}, J=$ $7.4 \mathrm{~Hz}, 1 \mathrm{H}, \mathrm{ArH}), 7.52$ (t, $J=7.7 \mathrm{~Hz}, 2 \mathrm{H}, \mathrm{ArH}), 4.29-4.14(\mathrm{~m}$, 2H, COOEt), 3.51 (dd, $J=9.7,7.4 \mathrm{~Hz}, 1 \mathrm{H}, \mathrm{CH}$-cyclo), 3.25 (s, $\left.3 \mathrm{H}, \mathrm{CH}_{3}\right), 2.38-2.25$ (m, 1H, $\mathrm{CH}_{2}$-cyclo), 2.17 (dd, $J=9.8,5.3$ $\mathrm{Hz}, 1 \mathrm{H}, \mathrm{CH}_{2}$-cyclo), 1.17 (t, $\left.J=7.1 \mathrm{~Hz}, 3 \mathrm{H}, \mathrm{COOEt}\right) .{ }^{13} \mathrm{C} \mathrm{NMR}$ (101 $\left.\mathrm{MHz}, \mathrm{CDCl}_{3}, 25{ }^{\circ} \mathrm{C}, \mathrm{TMS}\right): \delta=192.3,164.2,136.2,134.1$, 
$128.9,128.5,62.9,51.3,40.8,29.8,17.4,13.6$. IR (neat): $v$ 2984, 2935, 1730, 1682, 1597, 1581, 1450, 1372, 1312, 1229, 1161, 1129, 1097, 1019, 965, 929, 856, 779, $763 \mathrm{~cm}^{-1}$. HRMS (EI): $\mathrm{m} / \mathrm{z}$ calcd for $\mathrm{C}_{14} \mathrm{H}_{17} \mathrm{O}_{5} \mathrm{~S}[\mathrm{M}+\mathrm{H}]^{+}:$297.0797, found: 297.0793 .

\section{Acknowledgments}

This work was financially supported by grants from National Science Foundation of China (no. 21472053, 21172082). The Center of Analysis and Testing of Huazhong University of Science and Technology is gratefully acknowledged for characterization of the new compounds.

\section{References and notes}

1. For selected recent examples, see: (a) Fisher, L. A.; Smith, N. J.; Fox, J. M. J. Org. Chem. 2013, 78, 3342-3348; (b) Liu, Y.; Yu, Q.; Ma, S. M. Eur. J. Org. Chem. 2013, 3033-3040; (c) Young, P. C.; Hadfield, M. S.; Arrowsmith, L.; Macleod, K. M.; Mudd, R. J.; Jordan-Hore, J. A.; Lee, A. Org. Lett. 2012, 14, 898-901; (d) Liu, Y.; Ma, S. M. Org. Lett. 2012, 14, 720-723; (e) Miege, F.; Meyer, C.; Cossy, J. Chem. Eur. J. 2012, 18, 7810-7822; (f) Miege, F.; Meyer, C.; Cossy, J. Angew. Chem. 2011, 123, 6054; Angew. Chem. Int. Ed. 2011, 50, 5932-5937; (g) Liu, Y.; Ma, S. M. Chem. Sci. 2011, 2, 811-814; (h) Phan, D. H. T.; Kou, K. G. M.; Dong, V. M. J. Am. Chem. Soc. 2010, 132, 16354-16355; (i) Chen, S. J.; Ren, J.; Wang, Z. W. Tetrahedron. 2009, 65, 9146-9151; (j) Sherrill, W. M.; Rubin, M. J. Am. Chem. Soc. 2008, 130, 1380413809; (k) Alnasleh, B. K.; Sherrill, W. M.; Rubin, M. Org. Lett. 2008, 10, 3231-3234; (1) Lee, G. A.; Chen, K. C. Org. Lett. 2008, 10, 1843-1845; (m) Zhu, Z. B.; Shi, M. Chem. Eur. J. 2008, 14, 10219-10222; (n) Chuprakov, S.; D. Malyshev, A.; Trofimov, A.; Gevorgyan, V. J. Am. Chem. Soc. 2007, 129, 14868-14869; (o) Diev, V. V.; Stetsenko, O. N.; Tung, T. Q.; Kopf, J.; Kostikov, R. R.; Molchanov, A. P. J. Org. Chem. 2008, 73, 2396-2399; (p) Chuprakov, S.; Gevorgyan, V. Org. Lett. 2007, 9, 4463-4466; (q) Diev, V. V.; Kostikov, R. R.; Gleiter, R.; Molchanov, A. J. Org. Chem. 2006, 71, 4066-4077; (r) Yin, J. D.; Chisholm, J. D. Chem.
Commun. 2006, 632-634; (s) Pallerla, M. K.; Fox, J. M. Org. Lett. 2005, 7, 3593-3595; (t) Liao, L. A.; Yan, N.; Fox, J. M. Org. Lett. 2004, 6, 4937-4939; (u) Zohar, E.; Marek, I. Org. Lett. 2004, 6, 341-343; (v) Orugunty, R. S.; Ghiviriga, I.; Abboud, K. A.; Battiste, M. A.; Wright, D. L. J. Org. Chem. 2004, 69, 570-572; (w) Zohar, E.; Ram, M.; Marek, I. Synlett 2004, 1288-1290.

2. Zhang, M.; Gong, Y. F.; Wang, W. Z. Eur. J. Org. Chem. 2013, 7372-7381.

3. (a) Ochiai, M.; Kitagawa, Y.; Toyonari, M.; Uemura, K.; Oshima, K.; Shiro, M. J. Org. Chem. 1997, 62, 8001-8008; (b) Ochiai, M.; Kitogawa, Y.; Toyonari, M.; Uemurat, K. Tetrahedron letters 1994, 35, 9407-9408; (c) Ochiai, M.; Oshima, K.; Masaki, Y.; Kunishima, M.; Tanic, S. Tetrahedron letters 1993, 34, 48294830.

4. (a) Chang, Y. H.; Pinnick, H. W. J. Org. Chem. 1978, 43, 373374; (b) Corey, E. J.; Weatherhead-Kloster, R. A. Org. Lett. 2006, 8, 171-174.

5. (a) Baldwin, J. E.; Adlington, R. M.; Bebbington, D. Tetrahedron 1994, 50, 12015-12028; (b) Lai, M. T.; Oh, E.; Shih, Y.; Liu, H. W. J. Org. Chem. 1992, 57, 2471-2476.

6. Trost, B. M.; Cossy, J.; Burkes, J. J. Am. Chem. Soc. 1983, 105, 1052-1054.

7. Kazuta, Y.; Matsuda, A.; Shuto, S. J. Org. Chem. 2002, 67, 16691677.

8. Aissa, C. J. Org. Chem. 2006, 71, 360-363.

9. Bhaskar Reddy, D.; Ramana Reddy, P. V.; Padmavathi, V. Phosphorus, Sulfur, and Silicon 1994, 90, 1-10.

10. (a) Bhaskar Reddy, D.; Reddy, P. S.; Reddy, B. V.; Reddy, P. A. Synthsis 1987, 1, 74-75; (b) Padmaja, A.; Reddy, K. R.; Padmavathi, V.; Reddy, D. B. Collect. Czech. Chem. Commun. 1998, 63, 835-841. (c) Papageorgiou, C. D.; Ley, S. V.; Gaunt, M. J. Angew. Chem. Int. Ed. 2003, 42, 828-831.

11. (a) Balaji, T.; Bhaskar Reddy, D. Bulletin of the Chemical Society of Japan 1979, 52, 3434-3437; (b) Tanaka, K.; Suzuki, H. J. Chem. Soc. Perkin. Trans. 1992, 2071-2074.

12. Bray, C. D.; Faveri, G. D. J. Org. Chem. 2010, 75, 4652-4655.

13. (a) Meninno, S.; Parrella, A.; Brancatelli, G.; Geremia, S.; Gaeta, C.; Talotta, C.; Neri, P.; Lattanzi, A. Org. Lett. 2015, 17, 51005103; (b) Feng, J. B.; Gong, J. L.; Wu, X. F. RSC Adv. 2014, 4, 29273-29275; (c) Secci, F.; Frongia, A.; Piras, P. P. Tetrahedron Letters 2014, 55, 603-605.

Click here to remove instruction text... 


\section{Graphical Abstract}

To create your abstract, type over the instructions in the template box below.

Fonts or abstract dimensions should not be changed or altered.

\section{Highly Regioselective Tandem Formal}

Substitution and Decarboxylation of 2-Acyl-

1-Chlorocyclopropanecarboxylates with

Sodium Sulfinates

Yuequan Zhu, and Yuefa Gong*

School of Chemistry and Chemical Engineering, Huazhong University of Science and Technology, Wuhan 430074, People's Republic of China

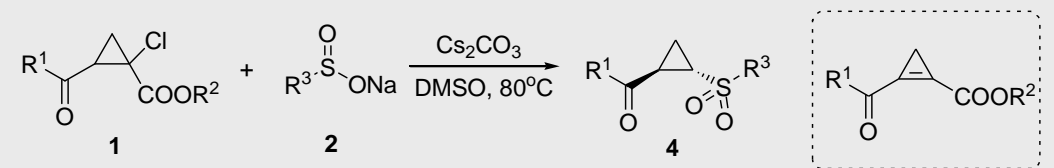

